Global arginine bioavailability ratio is decreased in patients with major depressive disorder

\title{
Ali-Sisto, Toni
}

2018-03-15

Ali-Sisto , T , Tolmunen , T , Viinamäki , H, Mäntyselkä , P, Valkonen-Korhonen , M , Koivumaa-Honkanen , H , Honkalampi , K, Ruusunen , A, Nandania , J , Velagapudi , V \& Lehto , S M 2018 , ' Global arginine bioavailability ratio is decreased in patients with major depressive disorder ' , Journal of Affective Disorders , vol. 229 , pp. 145-151 . https://doi.org/10.1016/j.jad.2017.12.0?

http://hdl.handle.net/10138/299679

https://doi.org/10.1016/j.jad.2017.12.030

publishedVersion

Downloaded from Helda, University of Helsinki institutional repository.

This is an electronic reprint of the original article.

This reprint may differ from the original in pagination and typographic detail.

Please cite the original version. 
Research paper

\title{
Global arginine bioavailability ratio is decreased in patients with major depressive disorder
}

\author{
Toni Ali-Sisto ${ }^{\mathrm{a}, *}$, Tommi Tolmunen ${ }^{\mathrm{b}}$, Heimo Viinamäki ${ }^{\mathrm{a}, \mathrm{b}}$, Pekka Mäntyselkäc ${ }^{\mathrm{c}}$, \\ Minna Valkonen-Korhonen ${ }^{\mathrm{a}, \mathrm{b}}$, Heli Koivumaa-Honkanen ${ }^{\mathrm{a}, \mathrm{b}, \mathrm{d}, \mathrm{i}, \mathrm{j}, \mathrm{k}}$, Kirsi Honkalampi ${ }^{\mathrm{e}}$, \\ Anu Ruusunen ${ }^{\mathrm{b}, \mathrm{f}}$, Jatin Nandania ${ }^{\mathrm{g}}$, Vidya Velagapudi ${ }^{\mathrm{g}}$, Soili M. Lehto ${ }^{\mathrm{a}, \mathrm{b}, \mathrm{h}}$ \\ a Institute of Clinical Medicine/Psychiatry, University of Eastern Finland, P.O. Box 1627, 70211 Kuopio, Finland \\ ${ }^{\mathrm{b}}$ Department of Psychiatry, Kuopio University Hospital, P.O. Box 100, 70029 KYS, Finland \\ ${ }^{c}$ Primary Health Care Unit, University of Eastern Finland and Kuopio University Hospital, P.O. Box 1627, 70211 Kuopio, Finland \\ ${ }^{\mathrm{d}}$ Departments of Psychiatry, South-Savonia Hospital District, Mikkeli, Finland \\ ${ }^{e}$ Department of Education and Psychology, University of Eastern Finland, P.O. Box 111, 80101 Joensuu, Finland \\ ${ }^{\mathrm{f}}$ Deakin University, Geelong, IMPACT Strategic Research Centre, VIC, Australia \\ ${ }^{\mathrm{g}}$ Metabolomics Unit, Institute for Molecular Medicine Finland FIMM, University of Helsinki, P.O. Box 20, 00014, Finland \\ ${ }^{\mathrm{h}}$ Department of Psychology and Logopedics, Faculty of Medicine, University of Helsinki, P.O. Box 9, 00014, Finland \\ i Departments of Psychiatry, North Karelia Central Hospital, Joensuu, Finland \\ ${ }^{\mathbf{j}}$ Departments of Psychiatry, SOTE, Iisalmi, Finland \\ ${ }^{\mathbf{k}}$ Departments of Psychiatry, Lapland Hospital District, Rovaniemi, Finland
}

\section{A R T I C L E I N F O}

\section{Keywords:}

Major depressive disorder

Arginine

Citrulline

Ornithine

Global arginine bioavailability ratio

\begin{abstract}
A B S T R A C T
Background: Major depressive disorder (MDD) is characterized by increased oxidative and nitrosative stress. We compared nitric oxide metabolism, i.e., the global arginine bioavailability ratio (GABR) and related serum amino acids, between MDD patients and non-depressed controls, and between remitted and non-remitted MDD patients.

Methods: Ninety-nine MDD patients and 253 non-depressed controls, aged 20-71 years, provided background data via questionnaires. Fasting serum samples were analyzed using ultra-performance liquid chromatography coupled to mass spectrometry to determine the serum levels of ornithine, arginine, citrulline, and symmetric and asymmetric dimethylarginine. GABR was calculated as arginine divided by the sum of ornithine plus citrulline. We compared the above measures between: 1$)$ MDD patients and controls, 2) remitted $(n=33)$ and non-remitted ( $\mathrm{n}=45$ ) MDD patients, and 3) baseline and follow-up within the remitted and non-remitted groups.

Results: Lower arginine levels (OR 0.98, 95\% CI 0.97-0.99) and lower GABR (OR 0.13, 95\% CI 0.03-0.50) were associated with the MDD vs. the non-depressed group after adjustments for potential confounders. The remitted group showed a decrease in GABR, arginine, and symmetric dimethylarginine, and an increase in ornithine after the follow-up compared with within-group baseline values. The non-remitted group displayed an increase in arginine and ornithine levels and a decrease in GABR. No significant differences were recorded between the remitted and non-remitted groups.

Limitations: The MDD group was not medication-free.

Conclusions: Arginine bioavailability may be decreased in MDD. This could impair the production of nitric oxide, and thus add to oxidative stress in the central nervous system.
\end{abstract}

\section{Introduction}

Major depression is the leading cause of disability and the global burden of disease, and is the most common psychiatric disorder in developed countries (WHO, 2014). However, the etiopathogenesis of major depressive disorder (MDD) remains poorly understood. Earlier studies have suggested aberrant nitric oxide (NO)-related pathways in MDD. In one earlier study, the bioavailability of arginine, a precursor of NO, was found to be decreased in inpatients with MDD (Baranyi et al., 2015). Furthermore, decreased activity of the endothelial isoform of nitric oxide synthase (eNOS) in platelets has been observed in MDD (Chrapko et al., 2006). However, first-episode MDD patients have been

\footnotetext{
* Correspondence to: Department of Psychiatry, Institute of Clinical Medicine, University of Eastern Finland, Finland

E-mail address: tonial@student.uef.fi (T. Ali-Sisto).
} 
shown to have increased levels of plasma nitrate, indicating increased production of NO (Suzuki et al., 2001).

Nitric oxide (NO) is gaseous signaling molecule, which is formed from the semi-essential amino acid arginine by three isoforms of nitric oxide synthases (NOS) (Magariños and McEwen, 1995). These isoforms are called inducible NOS (iNOS), neuronal NOS (nNOS), and endothelial NOS (eNOS). NO modulates vasodilation and neuronal functions, and inhibits the aggregation of platelets, adhesion of monocytes and leucocytes, proliferation of smooth muscle cells, oxidation of low density lipoprotein cholesterol (LDL), and vascular inflammation by suppressing chemokines and adhesion molecules (Böger et al., 1998; Cooke et al., 1991; Förstermann and Sessa, 2012; Tsao et al., 1996; Wolf et al., 1997). Inducible NOS (iNOS) is mainly expressed by macrophages in response to inflammatory signals. However, virtually any cell is capable of expressing iNOS (Förstermann and Sessa, 2012). In addition, NO decreases NMDA receptor activity (Lei et al., 1992; Manzoni and Bockaert, 1993).

As the half-life of NO is short and NO is mostly synthesized on demand at the site of need, there are no methods to reliably measure the levels of NO. Therefore, the only possibility is to measure the bioavailability of its precursor, which is also the limiting factor for the synthesis of NO. Previously, the capacity to synthetize NO has been estimated using the l-arginine/ornithine ratio, which reflects the activity of arginase, an enzyme that competes with NOS for arginine. However, the global arginine bioavailability ratio (GABR) is novel and improved approach used to measure the capacity of a system to produce NO, and has also been used as biomarker for endothelial dysfunction and cardiovascular risk factors (Sourij et al., 2011). GABR is calculated as the serum levels of arginine divided by the sum of ornithine plus citrulline. The ratio gives a more precise estimation of the bioavailability of arginine and thus the ability to produce NO (Morris et al., 2005; Romero et al., 2008a, 2008b; Sourij et al., 2011).

Asymmetric dimethylarginine (ADMA) is an endogenous competitive inhibitor of NOS (Vallance et al., 1992). Symmetric dimethylarginine (SDMA) has no NOS-inhibiting qualities, but has been thought to compete with the transportation of arginine through cell membranes (Leiper et al., 1999).

One recent study reported increased levels of ADMA and decreased levels of SDMA in patients with MDD (Baranyi et al., 2015).

In the present study, we analyzed the serum levels of arginine, citrulline, and ornithine, and calculated GABR for a longitudinal sample of MDD patients and for a non-depressed control group. Additionally, we analyzed the levels of asymmetric and symmetric dimethylarginine to assess the possible NOS-inhibiting role of these metabolites. These metabolites and GABR were compared 1) between the MDD and nondepressed groups and 2) within the MDD group between those who recovered and did not recover during the follow-up of eight months. Furthermore, we examined 3) changes in the metabolite levels during the follow-up period within the groups of a) remitted MDD patients and b) non-remitted MDD patients.

\section{Methods}

\subsection{Study samples}

The present study utilized two sample sets: 1) a naturalistic followup study sample of patients with MDD and 2) a general populationbased sample of non-depressed individuals (Ali-Sisto et al., 2016). The age distribution of the participants was 20-71 years. Both studies were approved by the Ethics Committee of Kuopio University Hospital. All participants provided written informed consent before entering the study. Both study samples geographically represent the same population.

The patient sample initially consisted of 100 outpatients with MDD, and was recruited from the Department of Psychiatry at Kuopio University Hospital. Due to a technical error during blood sample handling, one sample was unusable, and thus the final sample set consisted of 99 MDD patients. Due to the insignificant amount of missing data, no attrition analyses were performed. At baseline, the diagnosis of MDD was confirmed by using the Structured Clinical Interview for DSM-IV (SCID) (DSM-IV American Psychiatric Association, 1994). At the follow-up, the same criteria were used to confirm clinical depression or the remission status. Of the initial 99 patients, 78 participated in the follow-up study (mean follow-up time 8 months; range 5-13 months). We observed no differences in age ( $\mathrm{p}=$ $0.152)$, sex $(\mathrm{p}=0.663)$, marital status $(\mathrm{p}=0.575)$, alcohol use $(\mathrm{p}=$ 0.324), smoking $(\mathrm{p}=0.964)$, regular exercise $(\mathrm{p}=0.964)$, or BDI scores $(\mathrm{p}=0.493$ ) between the depressed patients who participated in the follow-up and those who did not. All participants gave venous blood samples at baseline and on follow-up. The exclusion criteria for the study were a history of epilepsy, bipolar disorder, psychotic disorders, mental symptomology due to substance abuse, and current somatic conditions preventing participation in the study.

At baseline, $84(84.8 \%)$ of the patients used antidepressant medication and $48(48.5 \%)$ used antipsychotic medication. Antidepressant use was distributed as follows: 1 ) selective serotonin reuptake inhibitors (SSRI), $\mathrm{n}=42$ (42.4\%); 2) venlafaxine, $\mathrm{n}=21$ (21.2\%); 3) mirtazapine, $\mathrm{n}=13$ (13.1\%); 4) duloxetine, $\mathrm{n}=12$ (12.1\%); 5) moclobemide, $\mathrm{n}=8(8.9 \%) ; 6)$ bupropion, $\mathrm{n}=6(6.1 \%)$, and 7) trazodone, $\mathrm{n}=1$ (1.0\%).

The non-depressed control sample was a population-based longitudinal cohort of 480 individuals living in the municipality of Lapinlahti, Finland. The sample included in this study was collected as part of the 5-year follow-up of the Lapinlahti Study in 2010. Altogether, 257 non-depressed controls were derived from the population-based Lapinlahti study sample. The exclusion criteria were an elevated level of depressive symptoms, i.e., Beck Depression Inventory (BDI) scores $\geq 10$ (Beck et al., 1961) at baseline or the 5-year follow-up of the Lapinlahti Study, or the reported use of antidepressants. Participants underwent a complete health examination and completed a background questionnaire (Savolainen et al., 2014).

\subsection{Background data}

The following variables were extracted from questionnaires completed by the participants in both study samples: the frequency of weekly physical exercise ( $\geq 1$ times vs. $<1$ time), regular smoking (yes vs. no), weekly alcohol use ( $0-5$ portions vs. $\geq 6$ portions; 1 portion corresponds to 1 bottle of beer, 1 glass of wine, or $4 \mathrm{cl}$ of spirits), marital status (married or living with a partner vs. living alone), educational level (university, polytechnic, or college education vs. lower than university, polytechnic, or college education), and a physiciandetermined diagnosis of hypertension (yes vs. no). Depressive symptoms were assessed with the 21-item BDI (range 0-63; Beck et al., 1961). The use of prescription and over-the-counter medications was also recorded with a questionnaire, and double-checked from the prescription documents the patients provided at the study visit.

\subsection{Laboratory analyses}

Before venipuncture, the participants in both study populations were instructed to fast for $12 \mathrm{~h}$. All samples were stored at $-70{ }^{\circ} \mathrm{C}$ until analyzed. The blood samples were used to quantify the concentrations of a batch of metabolites related to different aspects of the patient study and the population-based Lapinlahti Study, from which the non-depressed control sample was derived. Based on previous literature indicating a role of NO in MDD, this study focused on the precursor of NO, arginine, two closely related amino acids (i.e., ornithine and citrulline), and two metabolites know to regulate NOS enzymes (symmetric and asymmetric dimethylarginine).

Metabolites were extracted from the serum samples using acetonitrile (1:4, sample:solvent) and analyzed using a WATERS XEVO-TQ-S 
triple quadrupole mass spectrometer coupled to an ACQUITY UPLCMS/MS system (Waters Corporation, Milford, MA, USA). A detailed protocol and instrument conditions have been published elsewhere (Roman-Garcia et al., 2014). GABR was calculated as arginine divided by the sum of ornithine plus citrulline.

Serum high-density lipoprotein cholesterol (HDL-C; mmol/l) and glycated hemoglobin (HbA1c; \%) measurements were carried out according to the routine protocol in the accredited medical laboratory of Kuopio University Hospital. The analytical protocols have been described in detail elsewhere (Chang et al., 1998; Siekmann et al., 1976).

\subsection{Statistical methods}

To analyze the differences between groups, we used the chi-squared test and Fisher's exact test for categorical variables, and the MannWhitney $U$-test for continuous variables due to a non-normal distribution of the variables. The normality of the distribution for the continuous variables was examined using the Kolmogorov-Smirnov test. The changes in metabolite levels within the groups during the follow-up period were analyzed using the Wilcoxon signed-rank test. Correlations between the measured metabolites or GABR and variables related to the risk of cardiovascular morbidities were analyzed using Spearman's correlation coefficient or the Mann-Whitney $U$-test. Effect sizes for the analyzed group differences were calculated using an online calculator at http://www.socscistatistics.com/effectsize/Default3.aspx. Using the power of 0.80 and an alpha level of 0.05 , the study sample had statistical power to detect effects (Cohen's d) larger than 0.32 in the setting of MDD vs. non-depressed controls and 0.62 in the follow-up analyses (i.e., remitted vs. non-remitted MDD patients).

Logistic regression analysis (method: enter) was carried out. For baseline analyses, we constructed three models: the basic model (Model 1) was adjusted for age and gender. In the lifestyle model (Model 2), three factors (regular smoking, physical exercise, and alcohol use) were added to Model 1 to investigate the potential confounding effects of lifestyles factors. In the socioeconomic model (Model 3), education and marital status were added to Model 1. In the metabolic model (Model 4), serum levels of glycated hemoglobin (HbA1C) and fasting serum levels of high density lipoprotein cholesterol (HDL-C) were added to Model 2 to evaluate the potential confounding effect of metabolic status. Model 4 was constructed based on Model 2 rather than Model 3, because Model 2 showed a greater goodness-of-fit ( $-2 \log$ likelihood) compared to Model 3. The potential confounders were chosen based on a known influence of the examined variables (HbA1C and HDL-C, Peng et al., 2015; Zieliński and Kusy, 2015), or an observed difference between the investigated groups (regular smoking; physical exercise; alcohol use; see Table 1).

In the follow-up setting and in analyses comparing the remitted or non-remitted MDD groups and the non-depressed control group, a smaller number of participants $(\mathrm{n}=78)$ was available for analysis. Thus, fewer variables were utilized as covariates in the multivariate analysis in order to remain within the recommended $10 \%$ limit and avoid overfitting of the models (Babyak, 2004). Two models were tested. The basic follow-up model (Model 1) was identical to Model 1 used in the baseline setting, and was adjusted for age and gender. Follow-up Model 2 was further adjusted for physical exercise, as the level of physical exercise significantly differed between the remitted and non-remitted groups.

The possible confounding role of medication was assessed in two steps. First, the Mann-Whitney $U$-test was used to detect possible group differences in metabolite levels between MDD patients using and not using 1) any antidepressant, 2) SSRIs, 3) duloxetine, 4) venlafaxine, 5) mirtazapine, or 6) any antipsychotic. We did not examine the specific effects of bupropion or trazodone due to the small group sizes (bupropion, $\mathrm{n}=6$; trazodone, $\mathrm{n}=1$ ). Secondly, the possible confounding role of medications was further assessed by repeating the baseline multivariate Model 2 after the exclusion of individuals on any medication with significant metabolite differences between medication users vs. non-users. Model 4 was not applied to avoid over-adjusting the sample smaller following the exclusions (Babyak, 2004). All analyses were performed with SPSS 21.0 for Windows statistical software (SPSS Inc., Chicago, IL). Two-tailed p-values below 0.05 were considered to indicate statistical significance.

\section{Results}

\subsection{MDD patients vs. non-depressed controls at baseline}

The participants with MDD were younger than the non-depressed controls. Furthermore, they were more likely to smoke and less likely to exercise regularly (Table 1 ). GABR and the serum levels of arginine and symmetric dimethylarginine were lower in participants with MDD compared to non-depressed controls (Table 2). Effect sizes (Cohen's d) for group differences in the measured metabolites were 0.48 for GABR ( $\mathrm{p}<0.001), 0.68$ for arginine $(\mathrm{p}<0.001), 0.16$ for citrulline $(\mathrm{p}=$ $0.259), 0.08$ for ornithine ( $\mathrm{p}=0.460), 0.32$ for symmetric dimethylarginine $(\mathrm{p}=0.045)$, and 0.18 for asymmetric dimethylarginine $(\mathrm{p}=$ 0.460).

After fully adjusted logistic regression analysis (Model 4, Table 3), GABR and the serum levels of arginine remained significantly different between the participants with MDD and the non-depressed controls. Age and GABR correlated in the MDD group $(p=-0.281, p=0.005)$, but not in control group $(\rho=-0.058, p=0.355)$.

\subsection{Metabolites associated with MDD remission status at the follow-up}

At the time of the follow-up, 45 (57.7\%) patients still fulfilled the criteria for MDD, whereas $33(42.3 \%)$ had recovered. Non-remitted participants had higher baseline BDI scores and were less likely to exercise regularly than remitters (Table 1). Effect sizes (Cohen's d) for group differences in the measured metabolites were 0.08 for GABR ( $p$ $=0.836), 0.32$ for arginine $(\mathrm{p}=0.125), 0.24$ for citrulline $(\mathrm{p}=$ 0.259 ), 0.06 for ornithine ( $\mathrm{p}=0.421$ ), 0.17 for symmetric dimethylarginine $(\mathrm{p}=0.349)$, and 0.22 for asymmetric dimethylarginine ( $\mathrm{p}=$ 0.404). At the follow-up, remitted and non-remitted participants displayed no significant differences with regard to the investigated metabolites or GABR (Table 2). After fully adjusted logistic regression analysis (Model 4), no differences between groups emerged.

\subsection{Within-group changes in metabolite levels during the follow-up period}

During the follow-up period, MDD patients in the remitted group displayed increased GABR and levels of arginine and symmetric dimethylarginine, whereas the levels of ornithine decreased. In the nonremitted group, GABR and levels of arginine increased and levels of ornithine decreased over the follow-up period (Table 2, Table 4).

\subsection{Correlations between depressive symptoms and measured metabolites}

Within the MDD group, neither BDI scores nor the change in BDI scores correlated with any of the measured metabolites or GABR. Metabolite changes from baseline to the follow-up did not correlate with changes in BDI scores (Supplementary Table 1).

\subsection{Measured metabolites and variables affecting cardiovascular risk}

In the MDD group, those who had a diagnosis of high blood pressure $(\mathrm{n}=32)$ had lower GABR $(\mathrm{p}=0.003, \mathrm{Z}=-2.940)$ and higher levels of ornithine ( $\mathrm{p}=0.009, \mathrm{Z}=-1.855)$ than those without a diagnosis ( $\mathrm{n}=67$ ). However, HDL showed no correlation with the measured metabolites or GABR ( $p=0.307, \rho=-0.104)$. 
Table 1

Characteristics of the study groups at baseline. Values are medians (interquartile ranges) unless otherwise stated.

\begin{tabular}{|c|c|c|c|c|c|c|}
\hline & \multirow[t]{2}{*}{ MDD $n=99$} & \multirow{2}{*}{$\begin{array}{l}\text { Remitted } \\
\mathrm{n}=33\end{array}$} & \multirow{2}{*}{$\begin{array}{l}\text { Non-remitted } \\
\mathrm{n}=45\end{array}$} & \multirow{2}{*}{$\begin{array}{l}\text { Non-depressed } \\
\mathrm{n}=253\end{array}$} & \multicolumn{2}{|c|}{ Test statistics Test value (p-value) } \\
\hline & & & & & MDD vs controls & $\begin{array}{l}\text { Remitted vs } \\
\text { non-remitted }\end{array}$ \\
\hline Age, mean (SD) & $39.41(11.94)$ & $37.76(12.82)$ & $43.42(10.13)$ & $55.28(10.08)$ & $\mathrm{t}=12.60(<0.001)^{\mathrm{a}}$ & $\mathrm{t}=2.17(0.080)^{\mathrm{a}}$ \\
\hline Female, n (\%) & $56(56.6)$ & $17(51.5)$ & $28(62.2)$ & $129(51.0)$ & $\chi^{2}=0.89(0.346)^{\mathrm{b}}$ & $\chi^{2}=0.89(0.344)^{b}$ \\
\hline Married or living with a partner, $\mathrm{n}(\%)$ & $84(84.4)$ & $18(54.5)$ & $20(44.4)$ & $213(84.2)$ & $\chi^{2}=0.02(0.878)^{b}$ & $\chi^{2}=0.78(0.378)^{b}$ \\
\hline $\begin{array}{l}\text { University, polytechnic, or college } \\
\text { education, } \mathrm{n}(\%)\end{array}$ & $36(36.4)$ & $6(18.8)$ & $16(35.6)$ & $88(34.8)$ & $\chi^{2}=0.08(0.780)^{b}$ & $\chi^{2}=2.59(0.108)^{b}$ \\
\hline Regular smoking, n (\%) & $29(29.3)$ & $14(42.4)$ & $12(26.7)$ & $26(10.3)$ & $\begin{array}{l}\chi^{2}=19.52 \\
(<0.001)^{b}\end{array}$ & $\chi^{2}=2.13(0.145)^{b}$ \\
\hline Significant alcohol usage, n (\%) & $22(22.2)$ & $12(36.4)$ & $17(37.8)$ & $36(14.2)$ & $\chi^{2}=3.10(0.069)^{b}$ & $\chi^{2}=0.016(0.898)^{b}$ \\
\hline Regular exercise, n (\%) & $43(42.4)$ & $28(84.8)$ & $21(46.7)$ & $243(96.0)$ & $\begin{array}{l}\chi^{2}=132.77 \\
(<0.001)^{b}\end{array}$ & $\chi^{2}=11.88(0.001)^{b}$ \\
\hline HbA1C (\%) & $4.9(4.3-5.5)$ & - & - & $5.6(5.4-5.9)$ & $\begin{array}{l}Z=-8.02 \\
(<0.001)^{c}\end{array}$ & - \\
\hline HDL-C (mmol/l) & $1.0(0.7-1.5)$ & $1.56(1.19-1.99)$ & $1.39(1.15-1.83)$ & $1.4(1.1-1.7)$ & $\begin{array}{l}Z=-5.20 \\
(<0.001)^{c}\end{array}$ & $\mathrm{Z}=-1.03(0.302)^{\mathrm{c}}$ \\
\hline Diabetes, n (\%) & $5(5.1)$ & $1(3.0)$ & $3(6.7)$ & $21(8.2)$ & $\chi^{2}=1.10(0.295)^{b}$ & $\chi^{2}=0.52(0.472)^{b}$ \\
\hline Arterial hypertension, $\mathrm{n}(\%)$ & $32(32.3)$ & $6(18.2)$ & $19(42.2)$ & $78(30.8)$ & $\chi^{2}=0.07(0.786)^{b}$ & $\chi^{2}=5.05(0.025)^{b}$ \\
\hline Coronary artery disease, $\mathrm{n}(\%)$ & $3(3.0)$ & $1(3.0)$ & $2(4.4)$ & $7(2.8)$ & $\chi^{2}=0.18(0.894)^{b}$ & $\chi^{2}=0.10(0.748)^{b}$ \\
\hline Asthma, n (\%) & $12(12.1)$ & $2(6.1)$ & $7(15.6)$ & $22(8.6)$ & $\chi^{2}=0.95(0.328)^{b}$ & $\chi^{2}=1.68(0.195)^{b}$ \\
\hline BDI scores & $29(19.0-36.0)$ & $16(7-23)$ & $32(20-39)$ & $2.0(0.0-4.0)$ & $\begin{array}{l}Z=-14.12 \\
(<0.001)^{c}\end{array}$ & $Z=-5.05(<0.001)^{c}$ \\
\hline
\end{tabular}

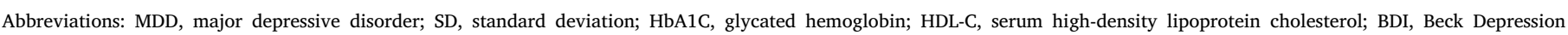
Inventory.

a Student's $t$-test.

${ }^{\mathrm{b}}$ Chi-squared test.

c Mann-Whitney $U$-test.

Table 2

Characteristics of the study groups. Values are medians (interquartile ranges).

\begin{tabular}{|c|c|c|c|c|c|c|c|}
\hline & & \multirow[t]{2}{*}{ MDD $n=99$} & \multirow[t]{2}{*}{ Remitted $\mathrm{n}=33$} & \multirow{2}{*}{$\begin{array}{l}\text { Non-remitted } \\
\mathrm{n}=45\end{array}$} & \multirow{2}{*}{$\begin{array}{l}\text { Non-depressed } \\
\mathrm{n}=253\end{array}$} & \multicolumn{2}{|c|}{ Test statistics ${ }^{\mathrm{a}}$ Test value (p-value) } \\
\hline & & & & & & $\begin{array}{l}\text { MDD vs non- } \\
\text { depressed }\end{array}$ & $\begin{array}{l}\text { Remitted vs } \\
\text { non-remitted }\end{array}$ \\
\hline \multirow[t]{3}{*}{ GABR } & Baseline & $0.86(0.63-1.08)$ & $0.75(0.61-1.07)$ & $0.84(0.62-1.10)$ & $1.01(0.79-1.28)$ & $\begin{array}{l}Z=-4.18 \\
(<0.001)\end{array}$ & $Z=-0.21(0.836)$ \\
\hline & Follow-up & - & $1.18(0.89-1.42)$ & $1.04(0.86-1.35)$ & - & - & $Z=-0.75(0.451)$ \\
\hline & Change & - & $0.42(0.06-0.57)$ & $0.23(-0.02-0.52)$ & - & - & $Z=-1.03(0.305)$ \\
\hline \multirow[t]{3}{*}{ Arginine } & Baseline & $99.15(83.1-115.92)$ & $\begin{array}{l}93.47 \\
(80.10-109.52)\end{array}$ & $\begin{array}{l}102.91 \\
(88.60-116.98)\end{array}$ & $\begin{array}{l}116.9 \\
(94.87-142.18)\end{array}$ & $\begin{array}{l}Z=-5.15 \\
(<0.001)\end{array}$ & $Z=-1.53(0.125)$ \\
\hline & Follow-up & - & $\begin{array}{l}109.89 \\
(98.13-135.92)\end{array}$ & $\begin{array}{l}120.25 \\
(99.12-139.75)\end{array}$ & - & - & $Z=-0.76(0.445)$ \\
\hline & Change & - & $\begin{array}{l}22.78 \\
(-2.97-35.96)\end{array}$ & $\begin{array}{l}13.56 \\
(-10.46-49.28)\end{array}$ & - & - & $Z=-0.54(0.588)$ \\
\hline \multirow[t]{3}{*}{ Citrulline } & Baseline & $29.01(22.01-33.69)$ & $29.06(19.84-33.38)$ & $29.27(24.00-36.49)$ & $29.44(23.31-36.61)$ & $\begin{array}{l}Z=-1.13 \\
(0.259)\end{array}$ & $Z=-0.93(0.355)$ \\
\hline & Follow-up & - & $27.41(22.37-33.46)$ & $30.45(24.24-40.41)$ & - & - & $Z=-1.78(0.076)$ \\
\hline & Change & - & $-0.6(-4.94-7.3)$ & $0.71(-5.44-8.86)$ & - & - & $Z=-0.76(0.445)$ \\
\hline \multirow[t]{3}{*}{ Ornithine } & Baseline & $\begin{array}{l}86.43 \\
(69.46-115.93)\end{array}$ & $\begin{array}{l}89.20 \\
(64.17-115.28)\end{array}$ & $90.16(76.71-122.46)$ & $\begin{array}{l}86.97 \\
(65.14-107.94)\end{array}$ & $\begin{array}{l}Z=-0.74 \\
(0.460)\end{array}$ & $\mathrm{Z}=-0.80(0.421)$ \\
\hline & Follow-up & - & $\begin{array}{l}79.96 \\
(51.07-101.13)\end{array}$ & $76.32(58.16-95.13)$ & - & - & $Z=-0.33(0.745)$ \\
\hline & Change & - & $\begin{array}{l}-16.72 \\
(-42.46-16.1)\end{array}$ & $\begin{array}{l}-16.54 \\
(-51.3-12.39)\end{array}$ & - & - & $Z=-0.15(0.883)$ \\
\hline \multirow[t]{3}{*}{$\begin{array}{l}\text { Symmetric } \\
\text { dimethylarginine }\end{array}$} & Baseline & $1.25(0.94-1.61)$ & $1.23(0.83-1.59)$ & $1.26(1.10-1.59)$ & $1.42(1.03-1.85)$ & $\begin{array}{l}Z=-2.00 \\
(0.045)\end{array}$ & $\mathrm{Z}=-1.00(0.349)$ \\
\hline & Follow-up & - & 1.37 (1.11-1.87) & $1.39(0.96-1.87)$ & - & - & $\mathrm{Z}=-0.36(0.720)$ \\
\hline & Change & - & $0.26(-0.42-0.84)$ & $0.05(-0.52-0.69)$ & - & - & $Z=-0.87(0.287)$ \\
\hline \multirow[t]{3}{*}{$\begin{array}{l}\text { Asymmetric } \\
\text { dimethylarginine }\end{array}$} & Baseline & $0.76(0.58-0.97)$ & $0.76(0.48-0.96)$ & $0.79(0.61-0.96)$ & $0.79(0.56-1.02)$ & $\begin{array}{l}Z=-0.74 \\
(0.460)\end{array}$ & $Z=-0.84(0.404)$ \\
\hline & Follow-up & - & $0.73(0.55-0.97)$ & $0.79(0.50-1.12)$ & - & - & $Z=-0.47(0.638)$ \\
\hline & Change & - & $0.13(-0.28-0.43)$ & $0.02(-0.39-0.49)$ & - & - & $Z=-0.33(0.742)$ \\
\hline
\end{tabular}

'Mann-Whitney $U$-test.

Abbreviations: MDD, major depressive disorder; GABR, global arginine bioavailability ratio. 
Table 3

Odds ratios (OR) and 95\% confidence intervals (CI) for the likelihood of belonging to the major depressive disorder group vs. the non-depressed group for each one-unit increase in the serum levels of each metabolite or the GABR.

\begin{tabular}{|c|c|c|c|c|}
\hline & & OR & $95 \%$ CI & $\mathrm{p}$ value \\
\hline \multirow[t]{5}{*}{ GABR } & Model 1 & 0.43 & $0.30-0.60$ & $<0.001$ \\
\hline & Model 2 & 0.53 & $0.35-0.81$ & 0.003 \\
\hline & Model 3 & 0.43 & $0.30-0.61$ & $<0.001$ \\
\hline & Model 4 & 0.51 & $0.32-0.80$ & 0.003 \\
\hline & Model $2^{\mathrm{a}}$ & 0.54 & $0.33-0.88$ & 0.014 \\
\hline \multirow[t]{5}{*}{ Arginine } & Model 1 & 0.52 & $0.37-0.74$ & $<0.001$ \\
\hline & Model 2 & 0.54 & $0.35-0.82$ & 0.004 \\
\hline & Model 3 & 0.52 & $0.37-0.73$ & $<0.001$ \\
\hline & Model 4 & 0.57 & $0.36-1.91$ & 0.018 \\
\hline & Model $2^{\mathrm{a}}$ & 0.48 & $0.28-0.83$ & 0.008 \\
\hline \multirow[t]{5}{*}{ Citrulline } & Model 1 & 1.25 & $0.93-1.67$ & 0.139 \\
\hline & Model 2 & 1.25 & $0.88-1.78$ & 0.217 \\
\hline & Model 3 & 1.24 & $0.92-1.67$ & 0.153 \\
\hline & Model 4 & 1.37 & $0.91-2.06$ & 0.128 \\
\hline & Model $2^{\mathrm{a}}$ & 1.10 & $0.72-1.68$ & 0.669 \\
\hline \multirow[t]{5}{*}{ Ornithine } & Model 1 & 1.39 & $1.05-1.84$ & 0.023 \\
\hline & Model 2 & 1.06 & $0.74-1.52$ & 0.760 \\
\hline & Model 3 & 1.38 & $1.04-1.83$ & 0.028 \\
\hline & Model 4 & 1.23 & $0.82-1.86$ & 0.319 \\
\hline & Model $2^{\mathrm{a}}$ & 0.98 & $0.65-1.45$ & 0.935 \\
\hline \multirow[t]{5}{*}{ Symmetric dimethylarginine } & Model 1 & 0.88 & $0.63-1.24$ & 0.472 \\
\hline & Model 2 & 0.83 & $0.53-1.31$ & 0.416 \\
\hline & Model 3 & 0.88 & $0.63-1.24$ & 0.474 \\
\hline & Model 4 & 0.84 & $0.52-1.36$ & 0.472 \\
\hline & Model $2^{\mathrm{a}}$ & 0.77 & $0.45-1.37$ & 0.379 \\
\hline \multirow[t]{5}{*}{ Asymmetric dimethylarginine } & Model 1 & 1.03 & $0.75-1.43$ & 0.842 \\
\hline & Model 2 & 1.04 & $0.67-1.58$ & 0.849 \\
\hline & Model 3 & 1.03 & $0.75-1.43$ & 0.836 \\
\hline & Model 4 & 1.06 & $0.67-1.68$ & 0.800 \\
\hline & Model $2^{\mathrm{a}}$ & 1.03 & $0.63-1.70$ & 0.900 \\
\hline
\end{tabular}

Model 1: Adjusted for age and gender.

Model 2: Model 1 further adjusted for regular smoking, significant alcohol use, and regular exercise.

Model 3: Model 1 further adjusted for university, polytechnic, or college education and cohabitation.

Model 4: Model 2 further adjusted for B-HbAC1 and high-density lipoprotein cholesterol. Abbreviations: OR, odds ratio; CI, confidence interval; GABR, global arginine bioavailability ratio.

${ }^{\mathrm{a}}$ Analyses with users of duloxetine, bupropion, and mirtazapine excluded.

Table 4

Longitudinal changes in metabolite levels from baseline to follow-up within the remitted and non-remitted MDD groups.

\begin{tabular}{lll}
\hline & $\begin{array}{l}\text { Remitted }(\mathrm{n}=33), \\
\mathrm{Z}(\mathrm{p} \text {-value })^{*}\end{array}$ & $\begin{array}{l}\text { Non-remitted } \\
(\mathrm{n}=45), \mathrm{Z}(\mathrm{p}-\mathrm{v}=\mathrm{lul})^{*}\end{array}$ \\
\hline GABR & $-3.53(\mathrm{p}<0.001)$ & $-3.00(\mathrm{p}=0.003)$ \\
Arginine & $-3.31(\mathrm{p}=0.001)$ & $-2.87(\mathrm{p}=0.004)$ \\
Citrulline & $-0.06(\mathrm{p}=0.950)$ & $-1.23(\mathrm{p}=0.221)$ \\
Ornithine & $-2.15(\mathrm{p}=0.031)$ & $-2.27(\mathrm{p}=0.023)$ \\
Symmetric dimethylarginine & $-2.99(\mathrm{p}=0.046)$ & $-1.03(\mathrm{p}=0.302)$ \\
Asymmetric dimethylarginine & $-0.81(\mathrm{p}=0.416)$ & $-0.29(\mathrm{p}=0.773)$ \\
\hline
\end{tabular}

Abbreviations: MDD, major depressive disorder; GABR, global arginine bioavailability ratio.

* Wilcoxon signed-rank test.

\subsection{The effect of medication use in the MDD sample}

In the MDD group, the use of duloxetine $(n=12)$ was associated with higher levels of citrulline ( $\mathrm{p}=0.034)$, the use of bupropion $(\mathrm{n}=$ $6)$ was associated with higher levels of arginine $(p=0.011)$ and ornithine ( $\mathrm{p}=0.033)$, and the use of mirtazapine $(\mathrm{n}=13)$ was associated with lower GABR ( $p=0.038$ ). The use of an SSRI, venlafaxine, any antidepressant medication (Table 5), or any antipsychotic medication did not associate with alterations in the metabolite levels. In multivariate Model 2 with users of duloxetine, bupropion, or mirtazapine excluded, the significant results were similar to the analyses in which users of these antidepressants were included (Table 3).

\section{Discussion}

\subsection{Summary of the main findings}

Firstly, we observed lower serum levels of arginine as well as a lower GABR in the MDD group compared to the control group at baseline. These findings persisted, regardless of adjustments for age, gender, alcohol use, regular smoking, physical exercise, HbA1C, and HDL-C. Secondly, at the follow-up, contrary to our hypotheses, no significant differences in the changes in metabolite levels were observed between remitted vs. non-remitted groups. Thirdly, within the remitted group, GABR and the levels of arginine and symmetric dimethylarginine increased over the follow-up period, whereas the levels of ornithine decreased. Within the non-remitted group, GABR and the levels of arginine increased, while the levels of ornithine decreased over the follow-up period. The increase in GABR was higher in the remitted group than in the non-remitted group.

\subsection{Comparison with the existing literature}

To the best of our knowledge, only two earlier studies have been published on GABR in psychiatric disorders (Baranyi et al., 2015; Bersani et al., 2016). Baranyi et al. (2015) investigated GABR, SDMA, ADMA, and the arginine/ADMA ratio in 71 inpatients suffering from MDD and 48 healthy controls. They found no difference in GABR between in-patients at the time of hospitalization and healthy controls. Furthermore, they observed elevated levels of ADMA and lowered levels of SDMA in MDD patients compared to controls. However, we observed a decreased GABR in MDD patients compared to controls and no differences in ADMA or SDMA in this study. Baranyi et al. recorded a non-significant increase in GABR during the time of hospitalization. In the present study, GABR significantly increased in both remitted and non-remitted groups during an average follow-up time of eight months. Compared to the present study, Baranyi et al. had a slightly smaller group size and investigated inpatients instead of outpatients. In general, inpatients tend to have either more severe or more acute symptoms than outpatients. Possibly, this could affect the differences in GABR or the measured metabolites between the study populations. However, our outpatient sample displayed slightly higher BDI scores (mean 29) compared to those of the inpatient sample of Baranyi et al. (mean 24). Moreover, Baranyi and co-workers utilized symptom scales instead of structured diagnostic methods. The present study was also able to assess potential confounders with multivariate models. Bersani et al. (2016) determined GABR in soldiers with and without posttraumatic stress disorder (PTSD), finding lower GABR in the participants with PTSD than the controls. Their results remained significant after adding diagnoses of MDD and antidepressant use as confounders to the multivariate model, suggesting an independent association between PTSD and GABR. Thus, decreased GABR may be a non-specific marker for several psychiatric disorders. In addition to psychiatric disorders, decreased GABR has been associated with increased cardiovascular morbidity and mortality from cardiovascular diseases (Sourij et al., 2011; Tang et al., 2009), which are both closely linked with depression (Penninx, 2016). In our sample, the depressed population was significantly younger compared to the non-depressed controls, and we also detected a correlation between age and GABR, but adjusting for age in the multivariate model did not affect our observations.

Baranyi et al. (2015) used solid phase extraction (SPE) and a derivatization method for the extraction of metabolites. Furthermore, they separated and detected the extracted metabolites by HPLC with a fluorescence detector. We used a one-step protein precipitation method for metabolite extraction. The extracted metabolites were detected using liquid chromatography-mass spectrometry, which is more 
Table 5

Characteristics of the users and non-users of antidepressants at baseline. Values are medians (interquartile ranges).

\begin{tabular}{|c|c|c|c|c|}
\hline & Any antidepressant $\mathrm{n}=84$ & No antidepressants $n=15$ & Test statistics ${ }^{\mathrm{c}}$ & p-value ${ }^{a}$ \\
\hline GABR & $0.86(0.65-1.13)$ & $0.87(0.60-1.01)$ & -0.77 & 0.441 \\
\hline Arginine & $101.31(85.72-116.36)$ & 85.99 (73.58-111.05) & -1.53 & 0.125 \\
\hline Ornithine & $86,21(68.65-117.31)$ & 96.58 (79.37-105.03) & -0.04 & 0.969 \\
\hline Citrulline & $29.11(22.06-34.22)$ & 28.80 (18.49-31.77) & -0.96 & 0.339 \\
\hline Symmetric dimethylarginine & $0.79(0.58-0.96)$ & $0.60(0.27-1.00)$ & -1.83 & 0.208 \\
\hline Asymmetric dimethylarginine & $1.26(0.97-2.62)$ & $1.11(0.56-1.57)$ & -1.26 & 0.068 \\
\hline
\end{tabular}

Abbreviations: GABR, global arginine bioavailability ratio.

${ }^{\text {a }}$ Mann-Whitney $U$-test (Z).

specific and sensitive compared to HPLC. We also used isotope-labelled internal standards to avoid a matrix effect during analysis in order to obtain optimized quantification. Finally, the metabolites were quantified from the area ratio (area of metabolites/area of internal standards (IS)) using calibration curves.

\subsection{Possible mechanisms}

Decreased GABR may indicate that the ability to produce NO is compromised or that the system has been producing NO in relatively large amounts to deplete the arginine supply. Increased NO production has been suggested to have a role in the etiopathogenesis of MDD. Suzuki et al. (2001) recorded increased plasma nitrate levels in MDD patients compared to patients with anxiety disorders, and found an increased production of NO.

INOS is activated as a response to inflammatory cytokines (e.g., interleukin-1 [IL] -1 , tumor necrosis factor [TNF]-alpha, and interferon gamma), and produces relatively large amounts of NO (Green et al., 1994; Knowles and Moncada, 1994). As MDD has been observed to be characterized by increased levels of proinflammatory cytokines (e.g. Valkanova et al., 2013), increased NO production induced by the pro-inflammatory state remains a fitting hypothesis for decreased GABR. MDD has been linked to a high state of oxidative and nitrosative stress, both of which are tied to inflammatory responses (Maes et al., 2011). Alternatively to or simultaneously with the possible increased activation of iNOS, decreased GABR could result from increased activity of the arginase enzyme. Arginase competes with NOS and converts arginine to ornithine, and is upregulated in conditions such as diabetes mellitus and inflammation (Morris et al., 2005; Maritza J. Romero et al., 2008a, 2008b).

MDD has been reported to be characterized by increased cardiovascular morbidity (Penninx, 2016). This association between depressive traits and cardiovascular events has been suggested to be explained by low-grade inflammation (Tolmunen et al., 2015). An inflammationinduced decrease in GABR might be one of the biological mechanisms behind the comorbidity of MDD and cardiovascular diseases. Decreased GABR has been reported to be a marker of increased cardiovascular mortality in patients hospitalized for coronary angiography (Sourij et al., 2011). In the present study, GABR was lower in MDD patients who had a diagnosis of hypertension compared to those patients with no diagnosis of hypertension.

If the ability to produce NO has been compromised due to decreased GABR, the neuronal functions of NO might also be compromised. In the central nervous system (CNS), nNOS produces NO and has generally been associated with synaptic plasticity, memory, long-term potentiation, and the regulation of blood pressure (Förstermann and Sessa, 2012). One of the known mechanisms of induction of NO production in the CNS is the activation of nNOS by an increased influx of Ca ions due to the activation of NMDA receptors (Bredt and Snyder, 1990; Weissman et al., 2002). The NMDA receptor has been studied as a pharmacological target for psychiatric treatments (Réus et al., 2016; Zorumski et al., 2015).

We did not observe significant group differences between the remitted and non-remitted MDD groups. Thus, it remains unclear how GABR changes during remission, and how quickly such effects might occur. The statistical power of the setting comparing remitted with nonremitted MDD patients was lower than that of the setting comparing MDD patients with non-depressed controls, and it is therefore possible that a larger sample comparing remitted with non-remitted MDD patients could detect differences in GABR. Nevertheless, it is possible that the differences observed between MDD patients and non-depressed controls in this study do not normalize during remission. Similar studies with larger group sizes and longer follow-up durations should be conducted to gain a better understanding of GABR kinetics during the remission phase of MDD.

\subsection{Strengths and limitations}

The strengths of the present study are the relatively large group size and the possibility to investigate the potential confounding effects of several possible biasing factors. Nevertheless, having a larger sample of depressed individuals might have allowed a more exact evaluation of potential differences in GABR between remitted and non-remitted MDD patients. Having access to a medication-free MDD sample would have allowed possible medication effects on NO metabolism to be excluded. Nevertheless, our observations remained unaltered after the exclusion of participants using medications that demonstrated associations with alterations in the investigated metabolites. Furthermore, the possibility to measure the activities of three isoforms of NOS and having access to more variables linked to cardiovascular morbidities would have further enhanced our analyses.

\section{Conclusions}

Major depressive disorder might be associated with the decreased bioavailability of arginine, which could impair the synthesis of nitric oxide.

\section{Acknowledgements}

The authors wish to thank Ms. Ritva von Koch, RN, for her assistance in the data collection.

\section{Role of the funding source}

The work of SML was supported by the Finnish Cultural Foundation. The funding source had no role in study design; in the collection, analysis and interpretation of data; in the writing of the report; and in the decision to submit the article for publication.

\section{Appendix A. Supporting information}

Supplementary data associated with this article can be found in the online version at http://dx.doi.org/10.1016/j.jad.2017.12.030. 


\section{References}

Ali-Sisto, T., Tolmunen, T., Toffol, E., Viinamäki, H., Mäntyselkä, P., Valkonen-Korhonen, M., Honkalampi, K., Ruusunen, A., Velagapudi, V., Lehto, S.M., 2016. Purine metabolism is dysregulated in patients with major depressive disorder. Psychoneuroendocrinology 70, 25-32. http://dx.doi.org/10.1016/j.psyneuen.2016. 04.017.

Babyak, M.A., 2004. What you see may not be what you get: a brief, nontechnical introduction to overfitting in regression-type models. Psychosom. Med. 66, 411-421.

Baranyi, A., Amouzadeh-Ghadikolai, O., Rothenhäusler, H.-B., Theokas, S., Robier, C. Baranyi, M., Koppitz, M., Reicht, G., Hlade, P., Meinitzer, A., 2015. Nitric oxiderelated biological pathways in patients with major depression. PLoS One 10 e0143397. http://dx.doi.org/10.1371/journal.pone.0143397.

Beck, A.T., Ward, C.H., Mendelson, M., Mock, J., Erbaugh, J., 1961. An inventory for measuring depression. Arch. Gen. Psychiatry 4, 561-571.

Bersani, F.S., Wolkowitz, O.M., Lindqvist, D., Yehuda, R., Flory, J., Bierer, L.M., Makotine, I., Abu-Amara, D., Coy, M., Reus, V.I., Epel, E.S., Marmar, C., Mellon, S.H., 2016. Global arginine bioavailability, a marker of nitric oxide synthetic capacity, is decreased in PTSD and correlated with symptom severity and markers of inflammation. Brain Behav. Immun. 52, 153-160. http://dx.doi.org/10.1016/j.bbi. 2015.10 .015$.

Bredt, D.S., Snyder, S.H., 1990. Isolation of nitric oxide synthetase, a calmodulin-requiring enzyme. Proc. Natl. Acad. Sci. U.S.A. 87, 682-685.

Böger, R.H., Bode-Böger, S.M., Kienke, S., Stan, A.C., Nafe, R., Frölich, J.C., 1998. Dietary L-arginine decreases myointimal cell proliferation and vascular monocyte accumulation in cholesterol-fed rabbits. Atherosclerosis 136, 67-77.

Chang, J., Hoke, C., Ettinger, B., Penerian, G., 1998. Evaluation and interference study of hemoglobin A1c measured by turbidimetric inhibition immunoassay. Am. J. Clin. Pathol. 109, 274-278.

Chrapko, W., Jurasz, P., Radomski, M.W., Archer, S.L., Newman, S.C., Baker, G., Lara, N., Le Mellédo, J.-M., 2006. Alteration of decreased plasma NO metabolites and platelet NO synthase activity by paroxetine in depressed patients. Neuropsychopharmacology 31, 1286-1293. http://dx.doi.org/10.1038/sj.npp.1300961.

Cooke, J.P., Rossitch, E., Andon, N.A., Loscalzo, J., Dzau, V.J., 1991. Flow activates an endothelial potassium channel to release an endogenous nitrovasodilator. J. Clin. Investig. 88, 1663-1671. http://dx.doi.org/10.1172/JCI115481.

Diagnostic and Statistical Manual of Mental Disorders (4th edn) (DSM-IV), 1994 Association, American Psychiatric, Washington, DC.

Förstermann, U., Sessa, W.C., 2012. Nitric oxide synthases: regulation and function. Eur. Heart J. 33, 829-837. http://dx.doi.org/10.1093/eurheartj/ehr304. (837a-837d).

Green, S.J., Scheller, L.F., Marletta, M.A., Seguin, M.C., Klotz, F.W., Slayter, M., Nelson, B.J., Nacy, C.A., 1994. Nitric oxide: cytokine-regulation of nitric oxide in host resistance to intracellular pathogens. Immunol. Lett. 43, 87-94.

Knowles, R.G., Moncada, S., 1994. Nitric oxide synthases in mammals. Biochem. J. 298 (Pt 2), 249-258.

Lei, S.Z., Pan, Z.H., Aggarwal, S.K., Chen, H.S., Hartman, J., Sucher, N.J., Lipton, S.A., 1992. Effect of nitric oxide production on the redox modulatory site of the NMDA receptor-channel complex. Neuron 8, 1087-1099.

Leiper, J.M., Santa Maria, J., Chubb, A., MacAllister, R.J., Charles, I.G., Whitley, G.S., Vallance, P., 1999. Identification of two human dimethylarginine dimethylaminohydrolases with distinct tissue distributions and homology with microbial arginine deiminases. Biochem. J. 343 (Pt 1), 209-214.

Maes, M., Galecki, P., Chang, Y.S., Berk, M., 2011. A review on the oxidative and nitrosative stress (O\&NS) pathways in major depression and their possible contribution to the (neuro)degenerative processes in that illness. Prog. Neuropsychopharmacol. Biol. Psychiatry 35, 676-692. http://dx.doi.org/10.1016/j.pnpbp.2010.05.004.

Magariños, A.M., McEwen, B.S., 1995. Stress-induced atrophy of apical dendrites of hippocampal CA3c neurons: involvement of glucocorticoid secretion and excitatory amino acid receptors. Neuroscience 69, 89-98. http://dx.doi.org/10.1016/03064522(95)00259-I.

Manzoni, O., Bockaert, J., 1993. Nitric oxide synthase activity endogenously modulates NMDA receptors. J. Neurochem. 61, 368-370.

Morris, C.R., Kato, G.J., Poljakovic, M., Wang, X., Blackwelder, W.C., Sachdev, V., Hazen, S.L., Vichinsky, E.P., Morris, S.M., Gladwin, M.T., 2005. Dysregulated arginine metabolism, hemolysis-associated pulmonary hypertension, and mortality in sickle cell disease. JAMA 294, 81-90. http://dx.doi.org/10.1001/jama.294.1.81.

Peng, T.-C., Wang, C.-C., Kao, T.-W., Chan, J.Y.-H., Yang, Y.-H., Chang, Y.-W., Chen, W.-
L., 2015. Relationship between hyperuricemia and lipid profiles in US adults. Biomed. Res. Int. 2015, 127596. http://dx.doi.org/10.1155/2015/127596.

Penninx, B.W.J.H., 2016. Depression and cardiovascular disease: epidemiological evidence on their linking mechanisms. Neurosci. Biobehav. Rev. http://dx.doi.org/10. 1016/j.neubiorev.2016.07.003.

Réus, G.Z., Abelaira, H.M., Tuon, T., Titus, S.E., Ignácio, Z.M., Rodrigues, A.L.S., Quevedo, J., 2016. Glutamatergic NMDA receptor as therapeutic target for depression. Adv. Protein Chem. Struct. Biol. 103, 169-202. http://dx.doi.org/10.1016/bs. apcsb.2015.10.003.

Roman-Garcia, P., Quiros-Gonzalez, I., Mottram, L., Lieben, L., Sharan, K., Wangwiwatsin, A., Tubio, J., Lewis, K., Wilkinson, D., Santhanam, B., Sarper, N., Clare, S., Vassiliou, G.S., Velagapudi, V.R., Dougan, G., Yadav, V.K., 2014. Vitamin $\mathrm{B}_{12}$-dependent taurine synthesis regulates growth and bone mass. J. Clin. Investig. 124, 2988-3002. http://dx.doi.org/10.1172/JCI72606.

Romero, M.J., Platt, D.H., Tawfik, H.E., Labazi, M., El-Remessy, A.B., Bartoli, M., Caldwell, R.B., Caldwell, R.W., 2008a. Diabetes-induced coronary vascular dysfunction involves increased arginase activity. Circ. Res. 102, 95-102. http://dx.doi.org/ 10.1161/CIRCRESAHA.107.155028.

Romero, M.J., Platt, D.H., Tawfik, H.E., Labazi, M., El-Remessy, A.B., Bartoli, M., Caldwell, R.B., Caldwell, R.W., 2008b. Diabetes-induced coronary vascular dysfunction involves increased arginase activity. Circ. Res. 102

Savolainen, J., Kautiainen, H., Miettola, J., Niskanen, L., Mäntyselkä, P., 2014. Low quality of life and depressive symptoms are connected with an unhealthy lifestyle. Scand. J. Public Health 42, 163-170. http://dx.doi.org/10.1177/ 1403494813504837.

Siekmann, L., Hüskes, K.P., Breuer, H., 1976. Determination of cholesterol in serum using mass fragmentography? A reference method in clinical chemistry. Fresenius' Z. für Anal. Chem. 279, 145-146. http://dx.doi.org/10.1007/BF00440813.

Sourij, H., Meinitzer, A., Pilz, S., Grammer, T.B., Winkelmann, B.R., Boehm, B.O., März, W., 2011. Arginine bioavailability ratios are associated with cardiovascular mortality in patients referred to coronary angiography. Atherosclerosis 218, 220-225. http:// dx.doi.org/10.1016/j.atherosclerosis.2011.04.041.

Suzuki, E., Yagi, G., Nakaki, T., Kanba, S., Asai, M., 2001. Elevated plasma nitrate levels in depressive states. J. Affect. Disord. http://dx.doi.org/10.1016/S0165-0327(00) 00164-6.

Tang, W.H.W., Wang, Z., Cho, L., Brennan, D.M., Hazen, S.L., 2009. Diminished global arginine bioavailability and increased arginine catabolism as metabolic profile of increased cardiovascular risk. J. Am. Coll. Cardiol. 53, 2061-2067. http://dx.doi. org/10.1016/j.jacc.2009.02.036.

Tolmunen, T., Lehto, S.M., Laukkanen, J., Ronkainen, K., Julkunen, J., Kauhanen, J., 2015. Somatic concerns, depressive traits, atherosclerosis and the incidence of cardiovascular disease in ageing Finnish men. J. Psychosom. Res. 79, 207-213. http:// dx.doi.org/10.1016/j.jpsychores.2015.05.006.

Tsao, P.S., Buitrago, R., Chan, J.R., Cooke, J.P., 1996. Fluid flow inhibits endothelial adhesiveness: nitric oxide and transcriptional regulation of VCAM-1. Circulation 94, 1682-1689. http://dx.doi.org/10.1161/01.CIR.94.7.1682.

Valkanova, V., Ebmeier, K.P., Allan, C.L., 2013. CRP, IL-6 and depression: a systematic review and meta-analysis of longitudinal studies. J. Affect. Disord. 150, 736-744. http://dx.doi.org/10.1016/j.jad.2013.06.004.

Vallance, P., Leone, A., Calver, A., Collier, J., Moncada, S., 1992. Accumulation of an endogenous inhibitor of nitric oxide synthesis in chronic renal failure. Lancet 339 , 572-575.

Weissman, B.A., Jones, C.L., Liu, Q., Gross, S.S., 2002. Activation and inactivation of neuronal nitric oxide synthase: characterization of $\mathrm{Ca} 2+$-dependent [125I]Calmodulin binding. Eur. J. Pharmacol. 435, 9-18. http://dx.doi.org/10. 1016/S0014-2999(01)01560-6.

WHO, 2014. Depression. Geneva.

Wolf, A., Zalpour, C., Theilmeier, G., Wang, B.Y., Ma, A., Anderson, B., Tsao, P.S., Cooke, J.P., 1997. Dietary L-arginine supplementation normalizes platelet aggregation in hypercholesterolemic humans. J. Am. Coll. Cardiol. 29, 479-485.

Zieliński, J., Kusy, K., 2015. Hypoxanthine: a universal metabolic indicator of training status in competitive sports. Exerc. Sport Sci. Rev. 43, 214-221. http://dx.doi.org/ 10.1249/JES.0000000000000055.

Zorumski, C.F., Nagele, P., Mennerick, S., Conway, C.R., 2015. Treatment-resistant major depression: rationale for NMDA receptors as targets and nitrous oxide as therapy. Front. Psychiatry 6, 172. http://dx.doi.org/10.3389/fpsyt.2015.00172. 